\title{
3. Marigranules from Glycine and Acidic, Basic, and Aromatic Amino Acids in a Modified Sea Medium
}

\author{
By Hiroshi Yanagawa and Fujio Egami \\ Mitsubishi-Kasei Institute of Life Sciences, 11 Minamiooya, \\ Machida-shi, Tokyo 194, Japan \\ (Communicated by Shiro Akabori, M. J. A., Jan. 12, 1978)
}

It is generally accepted that the origin of life took place in the primeval sea about $3 \times 10^{9}$ years ago and the essential components of living organisms must have been formed abiogenetically and accumulated in the sea by that time.

Egami found that a good correlation existed between the biological behavior of chemical elements and their concentrations in the contemporary sea water and presumed that transition elements relatively rich in sea water such as molybdenum, iron and zinc must have played important roles in the course of chemical evolution in primeval sea. ${ }^{1)}$

Based upon this consideration, we have been investigating the formation of biomolecules in modified sea mediums with lower concentration of sodium chloride and higher concentration of essential transition metal ions than in contemporary sea water and the formation of amino acids and related polymers from formaldehyde and hydroxylamine has been observed. ${ }^{2)}$ In the course of the research we observed that highly organized particles separated out after long incubation. The organized particles with guitar-like structures have been found to be produced with higher yields from glycine and formaldehyde in the same medium. ${ }^{3)}$ In the present paper we should like to describe the formation of spherical organized particles from glycine and acidic, basic, and aromatic amino acids in the modified sea medium. We propose to name organized particles produced in modified sea medium "marigranules."

Modified sea medium ( $\mathrm{ph}$ 5.2) contained $0.01 \mathrm{M}$ magnesium sulfate, $0.01 \mathrm{M}$ calcium chloride, $0.01 \mathrm{M}$ dibasic potassium phosphate and $0.1 \mathrm{mM}$ each of six transition metal ions $\left(\mathrm{Fe}\left(\mathrm{NO}_{3}\right)_{3}, \mathrm{Na}_{2} \mathrm{MoO}_{4}\right.$, $\mathrm{ZnCl}_{2}, \mathrm{Cu}\left(\mathrm{NO}_{3}\right)_{2}, \mathrm{CoCl}_{2}$, and $\mathrm{MnCl}_{2}$ ). Glycine (final concentration $0.05 \mathrm{M})$ and L-glutamic acid, L-aspartic acid, L-lysine, L-arginine, L-histidine, L-phenylalanine, L-tryptophan and L-tyrosine (each $5 \mathrm{mM}$ ) were added to the medium. The reaction mixture was glasssealed under nitrogen atmosphere and heated at $105^{\circ}$. In the course of reaction dark marigranules separated out and accumulated. After 
8 weeks the mixture was filtered on $0.2 \mu \mathrm{m}$ pore size of Nuclepore membrane. The precipitates were collected and suspended in a small amount of the filtrate. Purification of the marigranules were carried out with centrifugation in ascending concentration of Ficoll 400 (Mw, 400,000; 5-20\%). For scanning electron microscopy, the suspension of the marigranules was allowed to settle on clean glass coverslips, which were gently dried. Dried specimens were coated with platinum: palladium $(80: 20)$ in a JEE $4 \mathrm{~B}$ vacuum evaporator equipped with a rotary stage. Specimens were examined in a JEM 100C-ASID. Infrared spectrum of the purified marigranules was recorded on a JASCO IRA-1 grating instrument. Amino acids were analysed using a Hitachi KLA-5 automatic amino acid analyzer.

Fig. 1 shows ubiquitous marigranules observed with scanning electron microscopy. The marigranules had spherical structures of $0.3-2.5 \mu \mathrm{m}$ in diameter. Many of the marigranules possessed junctions. It seems likely that the junctions were not formed by binding each other but were produced by budding. Scanning electron micro-

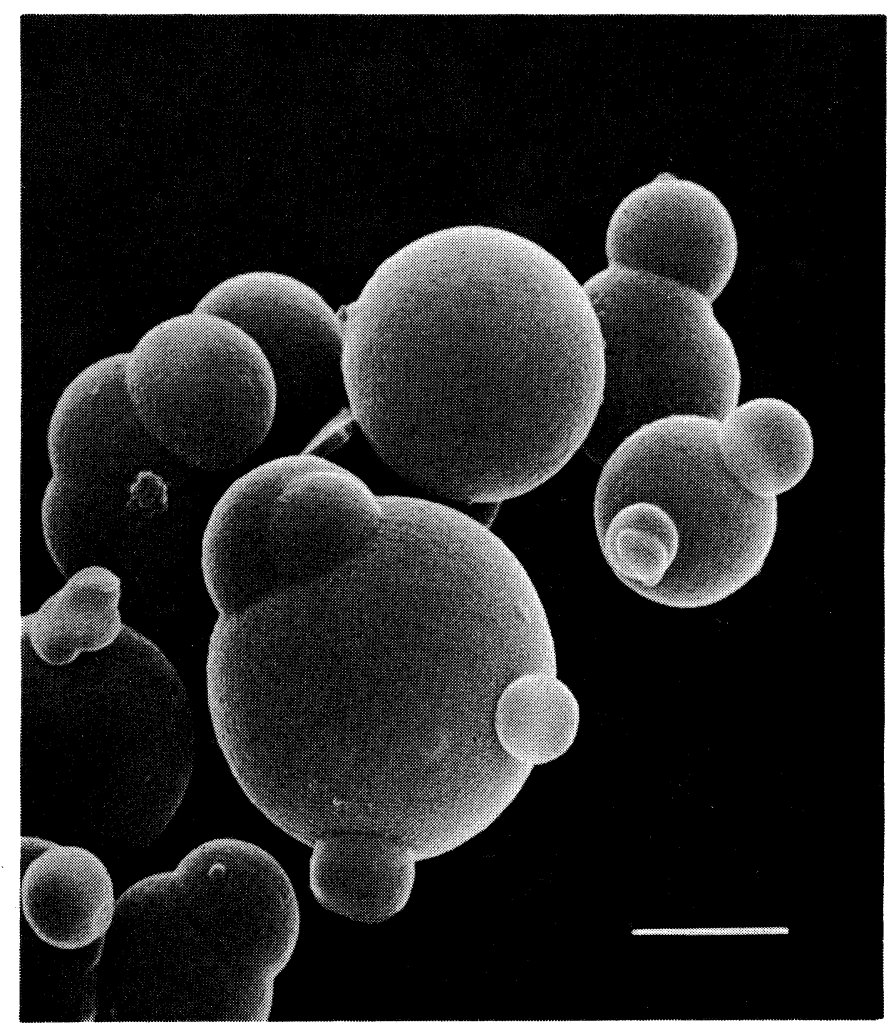

Fig. 1. Scanning electron micrograph of marigranules produced from glycine and acidic, basic and aromatic amino acids in a modified sea medium (scale bar, $1 \mu \mathrm{m}$ ). 
graph shows that the marigranules have four distinct surface structures (Fig. 2). Many of the particles possessed smooth surface structure (Fig. 3a), however, the occasional particles possessed rough surface structure (Fig. 3b), lightly fissured surface structures with small hollows of about $0.1 \mu \mathrm{m}$ in length (Fig. 3c), and surface structure with numerous small projections of about $800 \AA$ in diameter (Fig. 3d).

The purified marigranules had the percentage composition of carbon $58.22 \%$, hydrogen $3.76 \%$, nitrogen $14.23 \%$, ash $7.62 \%$, and others e.g., oxygen $16.17 \%$. The composition is somewhat similar to that of proteins. Infrared spectrum of the marigranules showed strong absorptions at 3560-2000, 1710, 1680-1610, 1550, 1510, 1450, $1380,1260,1160,1130,850$, and $750 \mathrm{~cm}^{-1}$. The infrared spectrum of the marigranules shows the presence of peptide bonds, i.e., first amide band (1680-1610 $\left.\mathrm{cm}^{-1}\right)$ and second amide band $\left(1550 \mathrm{~cm}^{-1}\right)$. Furthermore, by prolonged hydrolysis of the marigranules in $6 \mathrm{~N} \mathrm{HCl}$, relatively small amounts of glutamic acid, aspartic acid, phenylalanine,

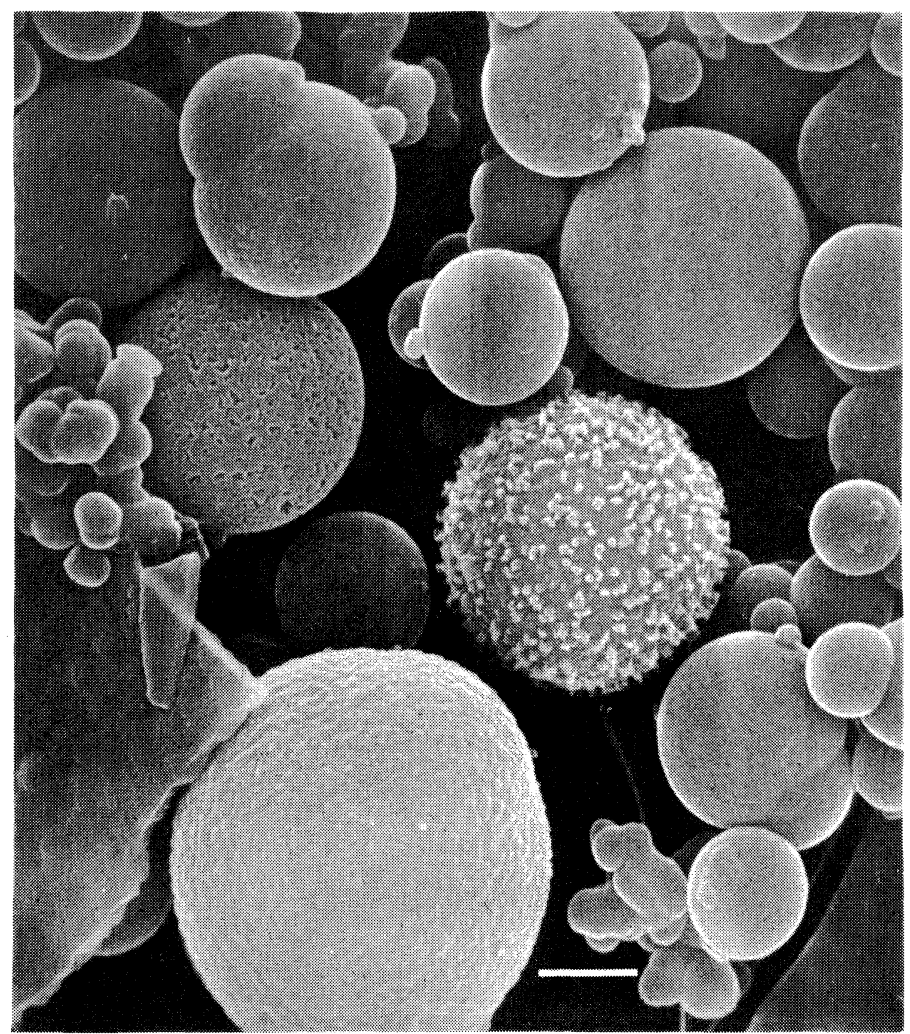

Fig. 2. Scanning electron micrograph of marigranules with different surface structures (scale bar, $1 \mu \mathrm{m}$ ). 

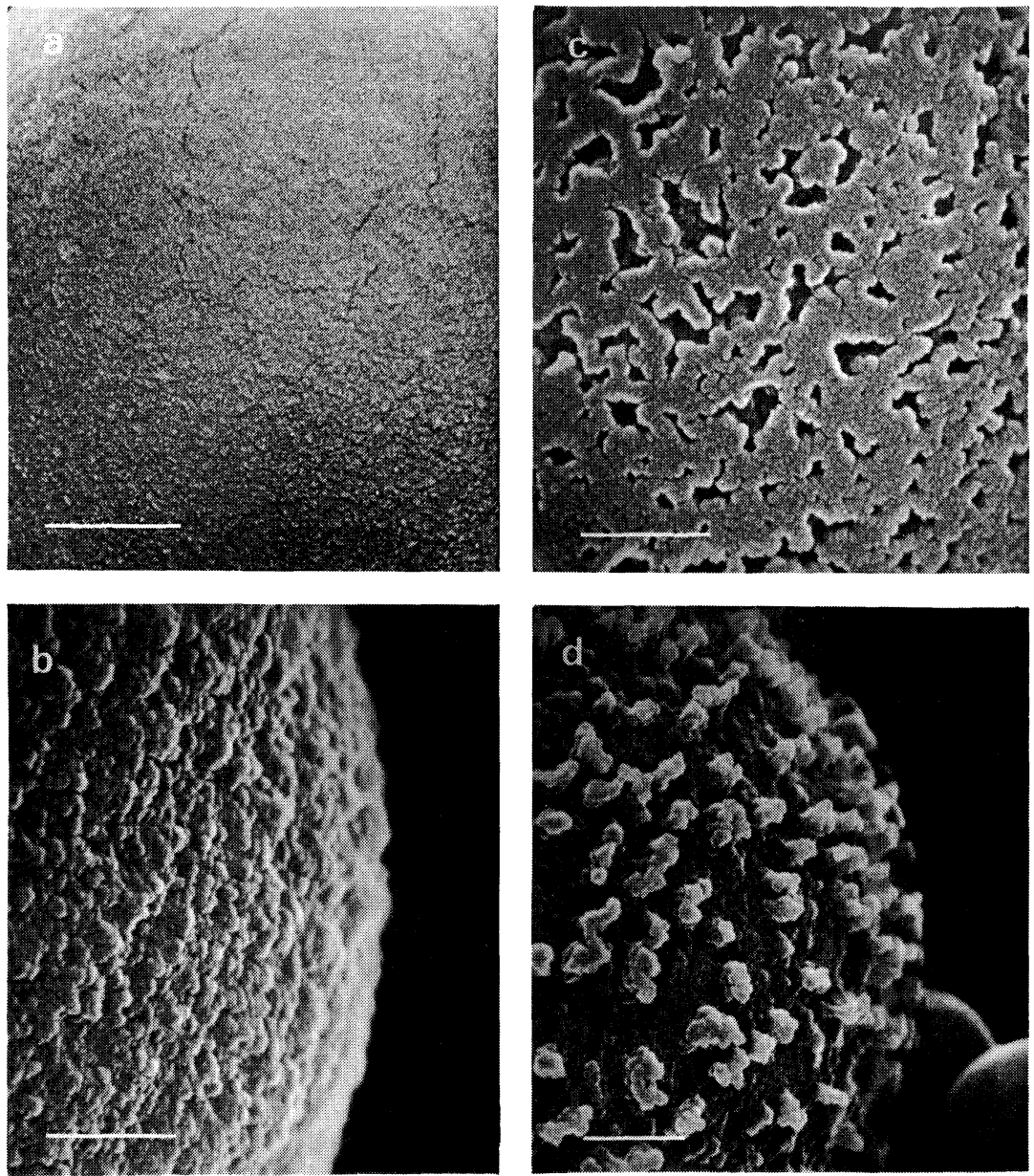

Fig. 3. Scanning electron micrographs of the surface structures of marigranules at higher magnifications (scale bars, $0.3 \mu \mathrm{m}$ ).

a, smooth surface structure. b, rough surface structure. c, lightly fissured surface structure with small hollows. d, surface structure with numerous small projections.

tyrosine, lysine, histidine, arginine, glycine, alanine, isoleucine, leucine, serine, threonine, and proline were detected. These observations may suggest the presence of extensively cross-linked melanoidin structures containing nitrogen heterocycles and polypeptidelike structures. The marigranules were completely solubilized with $1 \mathrm{~N} \mathrm{KOH}$ and the solubilized marigranules consisted of two main components with molecular weights of 45,000 and 7,900 daltons.

In conclusion highly organized particles named marigranules were produced from amino acids in a modified sea medium. It is suggested that such particles might be produced in primeval sea 
before the origin of life. Marigranules will join the group of protocell-like structures ${ }^{4)}$ such as coacervate droplets of Oparin and microspheres of Fox.

Acknowledgements. We thank Mr. S. Kondo and JEOL for electron microscopy and Drs. N. Murase and T. Ochiai for their valuable discussions.

\section{References}

1) Egami, F. (1974): J. Mol. Evol., 4, 113.

2) Hatanaka, H., and Egami, F. (1977): Bull. Chem. Soc. Japan, 50, 1147.

3) Yanagawa, H., and Egami, F. (1977): Proc. Japan Acad., 53, 42.

4) Fox, S. W. (1976): Origins of Life, 7, 49. 